\title{
Frame disruptions in human mRNA transcripts, and their relationship with splicing and protein structures
}

\section{Paul Harrison* and Zhan Yu}

Address: Department of Biology, McGill University, Stewart Biology Building, 1205 Docteur Penfield Ave., Montreal, QC, H3A 1B1 Canada

Email: Paul Harrison* - paul.harrison@mcgill.ca; Zhan Yu - zyu9@po-box.mcgill.ca

* Corresponding author

Published: 15 October 2007

BMC Genomics 2007, 8:37| doi:|0.||86/|47|-2|64-8-37|
Received: 3 April 2007

Accepted: 15 October 2007

This article is available from: http://www.biomedcentral.com/I47|-2/64/8/37।

(c) 2007 Harrison and Yu.; licensee BioMed Central Ltd.

This is an Open Access article distributed under the terms of the Creative Commons Attribution License (http://creativecommons.org/licenses/by/2.0), which permits unrestricted use, distribution, and reproduction in any medium, provided the original work is properly cited.

\begin{abstract}
Background: Efforts to gather genomic evidence for the processes of gene evolution are ongoing, and are closely coupled to improved gene annotation methods. Such annotation is complicated by the occurrence of disrupted mRNAs (dmRNAs), harbouring frameshifts and premature stop codons, which can be considered indicators of decay into pseudogenes.

Results: We have derived a procedure to annotate dmRNAs, and have applied it to human data. Subsequences are generated from parsing at key frame-disruption positions and are required to align significantly within any original protein homology. We find 419 high-quality human dmRNAs (3\% of total). Significant dmRNA subpopulations include: zinc-finger-containing transcription factors with long disrupted exons, and antisense homologies to distal genes. We analysed the distribution of initial frame disruptions in dmRNAs with respect to positions of: (i) protein domains, (ii) alternatively-spliced exons, and (iii) regions susceptible to nonsense-mediated decay (NMD). We find significant avoidance of protein-domain disruption (indicating a selection pressure for this), and highly significant overrepresentation of disruptions in alternatively-spliced exons, and 'nonNMD' regions. We do not find any evidence for evolution of novelty in protein structures through frameshifting.
\end{abstract}

Conclusion: Our results indicate largely negative selection pressures related to frame disruption during gene evolution.

\section{Background}

Mapping transcription information (mRNAs, cDNAs, ESTs, microarray data) onto the genomic DNA is an essential part of the gene annotation process. However, many transcripts appear to have frame disruptions in them, which would interfere with the formation of a stable protein product [1]. Such frame disruptions have generally been considered symptoms of decay into a pseudogene $[2,3]$.
Previously, we have analysed the distribution of a special case of such frame-disrupted transcripts, the 'transcribed processed pseudogene' or 'transcribed retropseudogene' [1]. Retropseudogenes are copies of messenger RNAs that have been reverse-transcribed and re-integrated into the genome, probably as a by-product of LINE-1 retrotransposition [4]. These intronless copies of genes usually decay and are deleted from the genomic DNA $[1,5-8,3]$. However, some retropseudogenes are transcribed, perhaps through co-option of local promoter elements, as supported by their increased density near genes $[2,9]$. 
Many mammalians genes ( $40-80 \%)$ make alternativelyspliced transcripts [10]. It has previously been noted that many such alternatively spliced exons (perhaps up to $\sim 30 \%$ ) harbour premature stop codons $[11,12]$, and thus may be considered 'pseudogenic' alternative transcripts. Other studies have demonstrated that several hundred human transcripts can be considered to harbour alternative reading frames, offset from each other by one or more frameshifts, which can be preserved for millions of years of evolution [13-15]. Frith, et al., found that about onetenth of mouse cDNAs have apparent frame disruptions [16]. Sorek, et al., showed that $\sim 7 \%$ of human genes generate an alternative transcript with an Alu, with the vast majority of these insertions yielding frame-disrupted mRNAs [17].

How frequent is genuine frame disruption in human mRNAs? Is it significantly associated with the positions of protein structures and exons in coding sequences? Here, to answer these and other questions, we analyze a data set of high-quality human mRNAs, using an annotation pipeline which insures that spurious frame disruptions (due simply to bad sequence alignment) are discarded. We perform statistical calculations which demonstrate non-random distribution of the initial frame disruptions in these sequences with respect to: (i) protein domain annotations, (ii) alternative exons and (iii) rules for nonsensemediated decay (NMD). Messenger RNA transcripts that have premature stop codons greater than fifty nucleotides $5^{\prime}$ to the last intron-exon junction of a gene are degraded by the nonsense-mediated decay (NMD) pathway [18]. Some NMD substrates have been shown to produce functional proteins in yeast and mammalian cells $[19,20]$. In addition, using our pipeline, we find no evidence for a role of frameshift in protein domain evolution.

\section{Results and discussion Overall statistics}

Using stringent thresholds, we verified 16,153 high-quality mRNAs from the NCBI Refseq and Unigene consensus collections, through mapping onto human genomic DNA. A small subpopulation of these $(419$, or $3 \%$ of the total) mRNAs harbour significant frame disruptions (either frameshifts or premature stop codons) (Table 1), which is of a similar order to previous analyses of such disruptions in sets of transcripts $[16,2,9]$. Most of these are disrupted by frameshifts ( $83 \%$ of cases), rather than premature stop codons. Using a small modification to the basic annotation pipeline, we defined a small minority of these frameshifted transcripts $(17,4 \%$ of the dmRNAs) that harbour compensating frameshifts, resulting in movement back into frame. Previous analysis of mouse cDNAs also indicated that a small fraction of them $(\sim 2 \%)$ may have such compensatory frameshifts [16]. Three examples of dmRNAs are illustrated in Figure 1. There are
Table I: Overall statistics

\begin{tabular}{ll}
\hline Data Set & Number \\
\hline $\begin{array}{ll}\text { Initial frame disruption is frameshift } \\
\text { Number with compensatory frameshifts }\end{array}$ & $346(83 \%)$ \\
Initial frame disruption is premature stop codon & $73(17 \%)$ \\
\hline Total & 419 \\
\hline
\end{tabular}

two multiply-disrupted examples (homologous to a cytochrome P450, and to a zinc-finger -containing transcription factor), and a frameshifted alternative mRNA transcript, from the gene C20orf59, which appears to be a transmembrane sugar transporter.

In general, the dmRNAs demonstrate functional prevalences that are typical of the population of human transcripts in general, as judged from counting up Gene Ontology functional category annotations (Additional File 1). The duplication behaviour of the genes from which the disrupted mRNAs arise is also typical of the whole human gene complement (Figure 2; median value of 5 paralogs per gene for the disrupted mRNAs versus 6 for the whole set; mean $=36[ \pm 62]$ versus $32[ \pm 81])$. However, dmRNAs have significantly fewer exons than mRNAs in general (mean $=7.9[ \pm 8.6]$ exons, compared to $10.0[ \pm$ 11.5] exons in general, $\mathrm{P}<0.05$ using normal statistics for the distribution of the sample mean). Such shorter lengths are expected from the truncating effect of frameshifts and stop codons. A large fraction (44\%) of the dmRNAs have multiple frame disruptions, with the frequencies of numbers of frame disruptions exhibiting a powerlaw relationship, as observed for processed pseudogenes $[7,8]$ (Figure 3). The vast majority of frameshifts in dmRNAs $(326 / 346,94 \%))$ result in truncation from premature stop codons.

We examined the etiology of the frame disruptions in dmRNAs in more detail. Some dmRNAs have apparent frame disruptions from $5^{\prime}$ and 3 ' insertions of retrotransposons $(24 / 325,7 \%)$, or from an overlapping antisense gene $(45 / 325,14 \%)$. Interestingly, also, a large proportion of dmRNAs arise from antisense homologies to other distal genes $(47 / 325,14 \%)$. Such antisense fragments are of potential importance in transcription regulation. A functional pseudogene with antisense homology to the nitric oxide synthase gene downregulates this gene the snail Lymnea stagnalis [21]. These three categories of dmRNA (retroposon insertions, antisense protein homologies and overlapping gene pair homologies) comprise a subset of dmRNAs arising from 'probable UTR (untranslated region) features'. Another possible source of dmRNAs are unassigned selenoproteins [22]. We have filtered for known selenoproteins [22], but it is possible there are 
(a) Multiple frame disruptions in a single-transcript gene

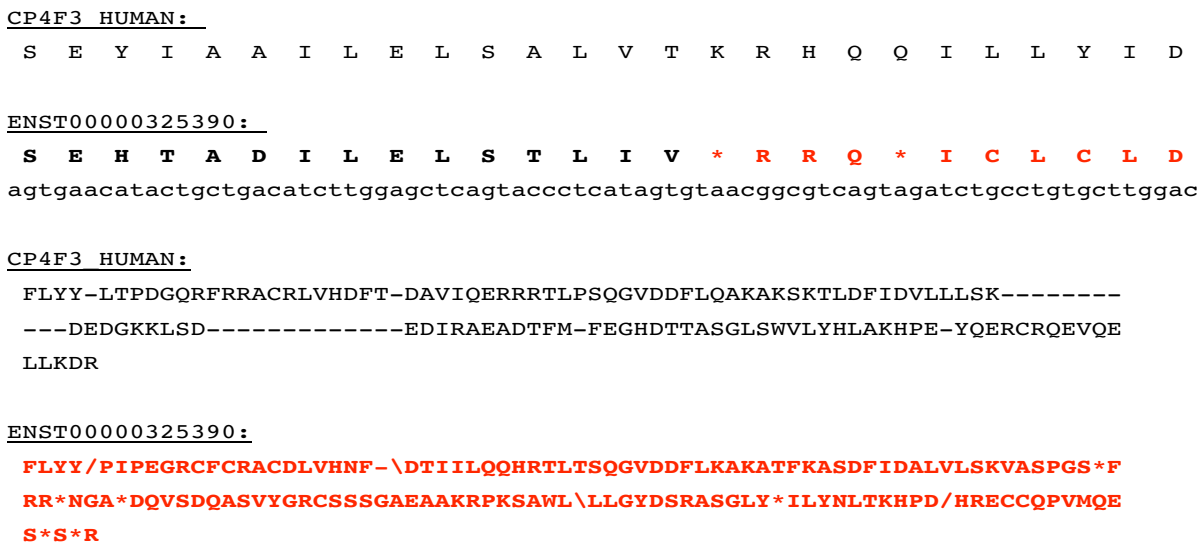

(c) Frame disruption from exon insertion

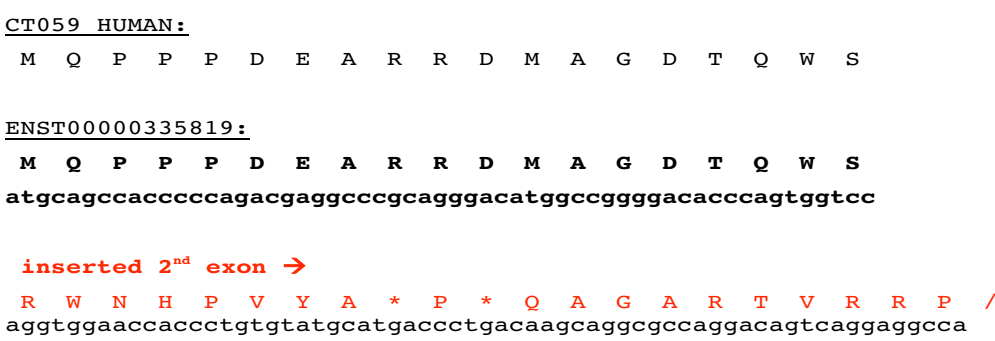

\section{Figure I}

Three examples of dmRNAs. The translated dmRNA sequence is shown along with the corresponding nucleotide sequence; the aligning protein sequence is shown above these in each case. They are as follows: (a) a multiply-disrupted example (homologous to a cytochrome P450); (b) a multiply-disrupted example from a zinc-finger -containing transcription factor family; (c) an alternative splicing of the transmembrane sugar transporter gene, C20orf59, which appears to be a transmembrane sugar transporter. 


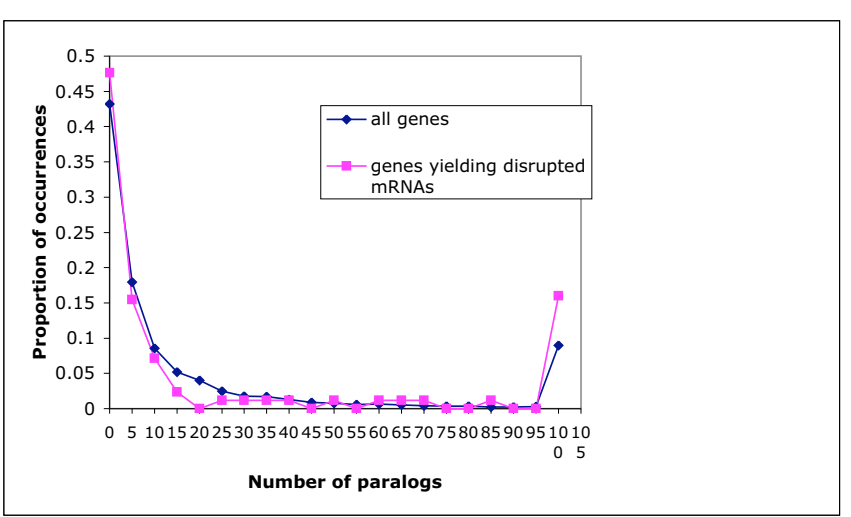

Figure 2

Numbers of paralogs. The distribution of the number of paralogs for all genes, and for genes yielding dmRNAs. The bin labeled $x$ contains all values $N$ such that $x-5<N \leq x$.

further cases. However, we found no indication of this, since there is no significant over-representation (relative to terminal stop codon frequencies in all Refseq mRNAs) of the opal stop codon (TGA) that is used for selenocysteine (38/57, $\mathrm{P}=0.12, \chi^{2}$ test, 2 degrees of freedom).

\section{Exon lengths}

Exons harbouring frame disruptions make up only a small fraction $(288 / 2432,12 \%)$ of the coding exons in their transcripts. Frame-disrupted exons are, on average, significantly longer $($ mean $=694$ nucleotides, compared to 248 nucleotides for undisrupted exons, $\mathrm{P}<0.001$ using normal statistics for the distribution of the sample mean). Although, both frame-disrupted and non-frame-disrupted exons show a tendency for very short exon lengths $(\leq 200$ nucleotides), there is a greater proportion of long exons ( $>1000$ nucleotides) in the frame-disrupted set $(24 \%$ of frame-disrupted exons versus $4 \%$ of those that are not frame-disrupted; Figure 4). To analyze exon lengths we disregarded the 'probable UTR features', but their inclusion does not change the trend observed; also, the exon length trend is maintained when exons are split into subsets of constitutive and alternatively-spliced exons. We examined the exons $>1000$ nucleotides in detail, and found that a significant fraction of them come from $\mathrm{Zn}$ finger -containing transcription factors $(36 / 67,54 \%)$ with $>1 / 3$ of their sequences composed of zinc finger motifs. Zinc-finger -containing transcription factors have dynamic evolution patterns in mammals, with expansions of family sizes specific to primates and rodents [23]; large numbers of dmRNAs are a signature of other dynamically evolving mammalian gene families, such as olfactory receptors and immune system genes [1]. A significantly greater proportion of disrupted exons are at the 3 ' terminus of mRNAs $(58 / 67,87 \%)$, even if the zincfinger -containing genes are excluded. Such 3 ' exons have

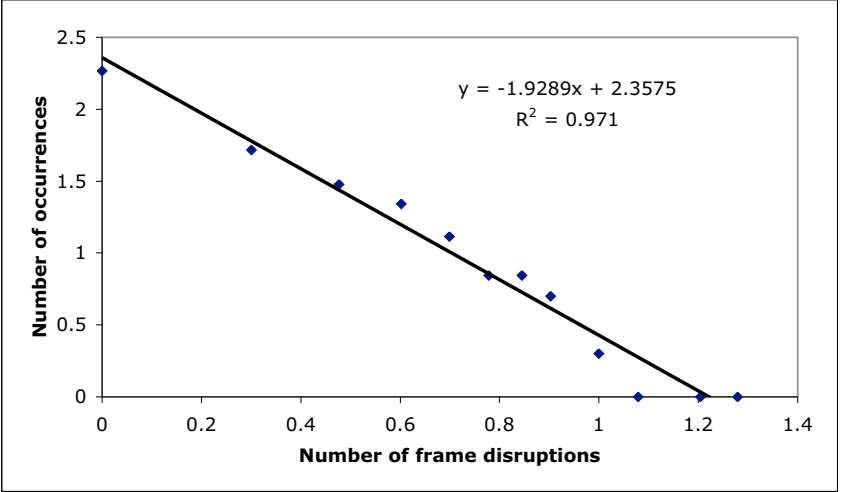

Figure 3

Numbers of frame disruptions. The number of frame disruptions in dmRNAs plotted versus the total occurrences of this number, on a log-log scale. This distribution is governed by a power law relationship, with the parameters for this linear relationship indicated on the plot.

a general tendency to be longer (51\% of 3' exons in multiple-exon transcripts verified by Refseq mRNAs are $\geq$ 1000 nucleotides in length) (Figure 4). This greater length has been suggested to be because of a greater amount of important conserved sequences in 3' UTRs, compared to 5' ones [24].

\section{Positions of frame disruptions in ImRNAs}

We analysed the distribution of the initial frame disruptions in the disrupted mRNAs with respect to the positioning of: (i) structural protein domains, (ii) alternativelyspliced exons, and (iii) the areas of the transcripts not susceptible to NMD (Tables 2, 3, 4, 5). In all of these analyses, we examine trends for the whole data set of dmRNAs, and the subset of these mRNAs for which the matching proteins have a verifying alignment in a divergent mammal or vertebrate (see Methods for details). The significant tendencies listed in Tables 2, 4 and 5 for the whole data set (combined stop codon and frameshift disruptions) remain significant or become more significant if those examples labelled 'probable UTR features' are removed from the data (this is illustrated for those with verifying alignments to divergent orthologs, in each case).

\section{Protein structure disruption}

Do the frame disruptions in these mRNAs avoid disruption of protein structure domains? To answer this question, we analysed the distribution of initial frame disruptions in sequences relative to the placement of protein structure domains from the SCOP data (see Methods for details). For both frameshifts and premature stop codons, we find significant underrepresentation within protein domains ( $\mathrm{P}$ in range $<0.05$ to $<0.001$; all of the $\mathrm{P}$ values quoted for Tables $2,3,4,5$ are for $\chi^{2}$ tests; see Table 

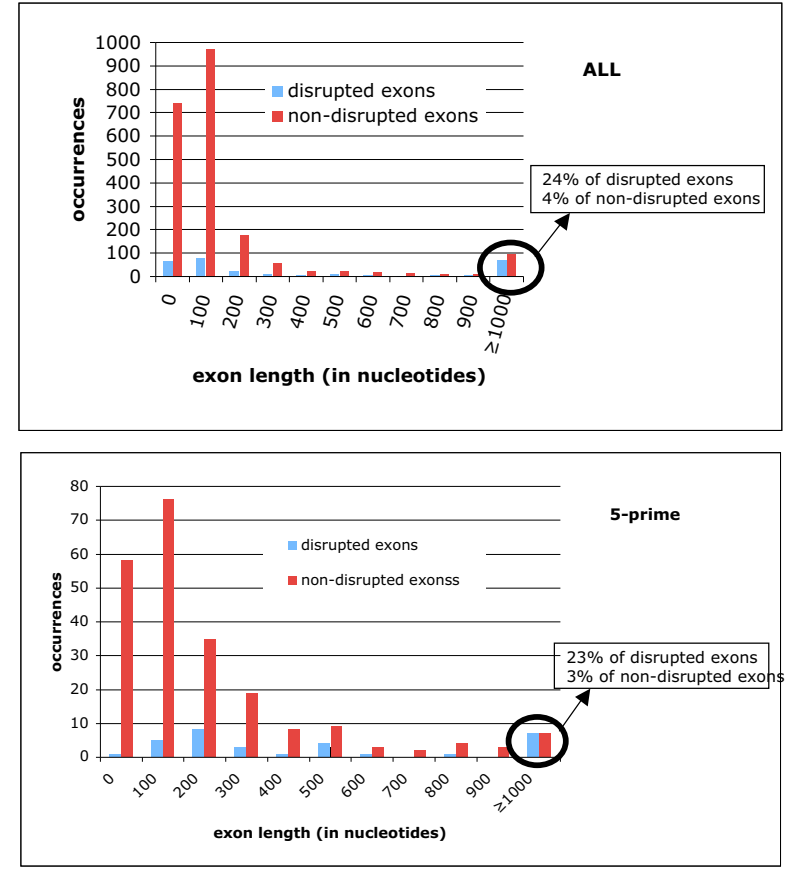
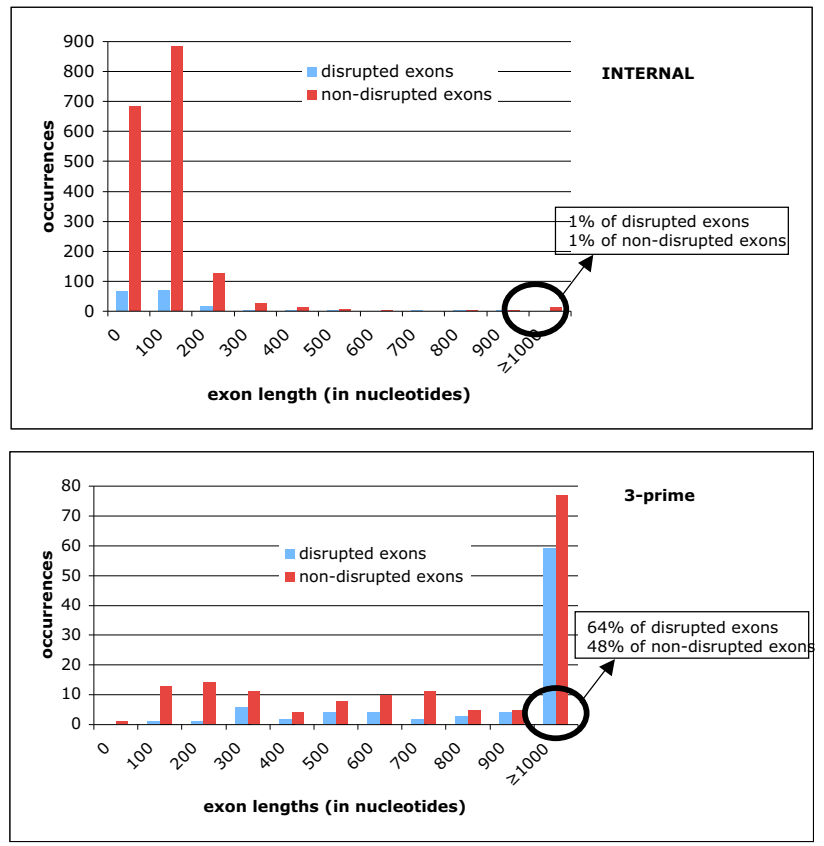

\section{Figure 4}

Distribution of frame-disrupted and non-frame-disrupted exon lengths in the disrupted mRNAs. The exon lengths are in bins labelled at either end of the bin with the upper $(\leq)$ and lower $(>)$ bounds, with occurrences in each bin on the $y$ axis. The percentage of exons $>1000$ nucleotides is given for each data set. The upper left panel is for the whole set of exons; the lower left panel for 5' exons, the upper right for internal exons, and the lower right for 3' exons.

2 footnote for details of statistical tests). This non-random distribution of frame disruptions is observed for a wide range of margins for definition of overlap with protein domains (between 0 and 25 nucleotides) (Table 2 footnote). This avoidance of protein structure domains is evidence for selection pressures to avoid protein structure

Table 2: Protein structure disruptions in mammalian messenger RNA transcripts

\begin{tabular}{|c|c|c|c|c|}
\hline \multirow[t]{2}{*}{$\begin{array}{l}\text { Type of initial } \\
\text { frame disruption* }\end{array}$} & \multirow[t]{2}{*}{ Alignment verification $* *$} & \multicolumn{3}{|l|}{$\begin{array}{l}\text { Protein structure } \\
\text { disruption*** }\end{array}$} \\
\hline & & $\begin{array}{l}\text { Observed } \\
\text { [nondisrupting : disrupting] }\end{array}$ & $\begin{array}{l}\text { Expected } \\
\text { [nondisrupting : disrupting] }\end{array}$ & Significance \\
\hline \multirow[t]{2}{*}{ Frameshift } & All cases & $293: 54$ & $272.7: 74.3$ & $+\dagger$ \\
\hline & Cases with verifying alignments & $230: 51$ & $211.3: 69.7$ & ttt \\
\hline \multirow[t]{2}{*}{ Stop codon } & All cases & $68: 5$ & $55.6: 17.4$ & tt† \\
\hline & Cases with verifying alignments & $34: 5$ & $27.4: 11.6$ & $\dagger$ \\
\hline \multirow[t]{3}{*}{ Frameshift or stop codon } & All cases & $360: 59$ & $327.5: 91.5$ & $t+\dagger$ \\
\hline & Cases with verifying alignments & $268: 57$ & $242.2: 82.8$ & t† \\
\hline & $\begin{array}{l}\text { Cases with verifying alignments } \\
\text { (excluding probable UTR features) }\end{array}$ & $174: 35$ & $153.6: 55.4$ & ttt \\
\hline
\end{tabular}

* For the last row, those with frameshifts and stop codons are pooled together.

*** Verifiying alignments are significant alignments to a rodent or non-mammalian vertebrate protein, as detailed in Methods.

*** The ratio stands for 'the number of frame disruptions not disrupting a protein structure domain assignment versus the number that do'. A margin for ascertaining overlap with a protein domain assignment of 15 nucleotides was used in the calculations. The expectations for the statistical tests $\left(\chi^{2}\right)$ are calculated by adding up the total amount of coding sequence that can be assigned to a SCOP protein structure domain for the sample of transcripts analysed in each row of the table. $\dagger$ stands for $P<0.05$, $\dagger \dagger$ for $P<0.01$ and $\dagger \dagger \dagger$ for $P<0.00$ I. The significant results remain significant to at least $P<0.05$ when margins for calculating overlap with protein domains of $0,5,10,20$ or 25 nucleotides are also used. 
Table 3: Distribution of initial disablements relative to zinc-finger domains *

\begin{tabular}{cccc}
\hline Margin & Observed [inside domain: outside domain] & Expected [inside domain: outside domain] & Significance \\
\hline 0 & $21: 20$ & $26.7: 14.3$ & N.S. \\
1 & $17: 24$ & $24.7: 16.3$ & $\dagger$ \\
2 & $14: 21$ & $22.1: 18.9$ & $\dagger$ \\
3 & $12: 29$ & $19.7: 21.3$ & + \\
4 & $10: 31$ & $17.3: 23.7$ & $\dagger$ \\
5 & $10: 31$ & $14.9: 26.1$ & N.S. \\
\hline
\end{tabular}

* The format of this Table is as for Tables 2-3. The overlap margin is the number of residues that are ignored at either end of the zinc-finger domain (thus shortening the length of the defined protein motif).

disruption and supports a significantly negative role for frame disruption in the evolution of protein structures.

Because of the proportion of dmRNAs that contain large arrays of $\mathrm{Zn}$ finger domains, we also checked specifically for avoidance of disruption of $\mathrm{Zn}$ finger motifs. Zn finger motif assignments were taken from the feature table records of the Uniprot database [25]. We find significant avoidance of disruption of $\mathrm{Zn}$ finger motifs only for overlap margins of between 1 and 4 residues inclusive (Table 3).

\section{Alternative splicing}

We examined whether there is a relationship between the position of initial frame disruptions in mRNAs and the location of alternatively spliced exons (Table 4). We find a highly significant two-fold overrepresentation of initial frame disruptions in alternatively-spliced exons $(P<$ 0.001 ; Table 4). These correspond to almost half ( $46 \%$, $191 / 419)$ of the dmRNAs. This may arise because the selection pressure on alternative splicings that are not transcribed at high levels will be considerably less, leading to increased likelihood of frame disruption as evolution progresses [10]. It is possible that many of these framedisrupted alternative splicings have a regulatory role $[11,26]$. Small numbers of the alternatively-spliced frameshifted dmRNAs arise from exon skipping (4 cases), and exon insertion (21 cases). This approximately twofold over-representation is maintained $(\mathrm{P}<0.05)$ in the subset of alternative splicings that contain SCOP [27] protein domain assignments within them.

\section{Transcripts not susceptible to nonsense-mediate decay}

Messenger RNA transcripts that have premature stop codons greater than fifty nucleotides 5 ' to the last intronexon junction of a gene are degraded by nonsense-mediated decay (NMD) [18]. We analyzed the distribution of initial frame disruptions relative to this NMD rule (Table $5)$. There are significantly more transcripts with frame disruptions in the 'non-NMD' region $(\mathrm{P}<0.001$; Table 5$)$, as would be expected logically (since these transcripts would not be degraded). However, this over-representation of initial frame disruptions in the 'non-NMD' region also arises for the subsets of transcripts in which the frame disruptions disrupt a SCOP protein structure domain $(\mathrm{P}<$ $0.05)$, and which are thus unlikely to form a stable func-

Table 4: Frame disruption placement and alternative splicing

\begin{tabular}{|c|c|c|c|c|}
\hline \multirow{2}{*}{$\begin{array}{l}\text { Type of initial frame } \\
\text { disruption }\end{array}$} & \multirow[t]{2}{*}{ Alignment verification } & \multicolumn{3}{|l|}{ Type of exon * } \\
\hline & & $\begin{array}{l}\text { Observed } \\
\text { [constitutive : alternative] }\end{array}$ & $\begin{array}{l}\text { Expected } \\
\text { [constitutive : alternative] }\end{array}$ & Significance \\
\hline \multirow[t]{2}{*}{ Frameshift } & All cases & $191: 156$ & $258.8: 88.2$ & t+t \\
\hline & Cases with verifying alignments & $156: 125$ & $209.6: 71.4$ & ttt \\
\hline \multirow[t]{2}{*}{ Stop codon } & All cases & $33: 40$ & $54.4: 18.6$ & ttt \\
\hline & Cases with verifying alignments & $13: 26$ & $29.1: 9.9$ & ttt \\
\hline \multirow[t]{3}{*}{ Frameshift or stop codon } & All cases & $228: 191$ & $312.5: 106.5$ & t†t \\
\hline & Cases with verifying alignments & $|74:| 5 \mid$ & $242.4: 82.7$ & ttt \\
\hline & $\begin{array}{l}\text { Cases with verifying alignments } \\
\text { (without 'probable UTR features') }\end{array}$ & $114: 95$ & $143.8: 65.2$ & ttt \\
\hline
\end{tabular}

* Ratios are for numbers expected or observed in constitutive exons versus alternative ones. Expectations and test are performed as for Table I. 
tional protein product. Such unstable protein products are more likely for shorter truncations, and thus NMD provides an evolutionary guard against excessive expression of unstable proteins [28].

\section{Checking for gene evolution through frame-shift formation} It is possible that through analysis of this comprehensive data set of dmRNAs, that we can find evidence for a positive role for such protein-coding frame disruptions in gene evolution. Specifically, is there evidence that such frameshifts can produce significant structural novelties? To check this, we derived a modification for the initial pipeline (Figure 5), with matches to SCOP protein structure domains replacing those for whole protein sequences from the SWISSPROT database, finding 36 cases ( $9 \%$ of the dmRNAs) which produce a significant alignment for both subsequences delimited by the initial frameshift (Figure 5, step 3). However, none of these (0\%) overlap another protein domain assignment in a different frame, yielding no evidence for generation of protein structure novelties through single frameshifts. Nonetheless, a more thorough analysis of multiple vertebrates would be required to provide a more conclusive perspective on the role of frameshift in protein structure evolution.

\section{Conclusion}

We analyzed human mRNAs for both frameshift and stop codon frame disruptions, using a pipeline that was designed to discard spurious frame disruptions arising from alignment error. We performed statistical calculations and found non-random distributions of frame disruptions with respect to protein structures, alternativelyspliced exons, and 'non-NMD' regions. The significant avoidance of protein structure disruption and highly significant placement in alternatively-spliced exons (rather than constitutive ones), together with the observation of a lack of protein structure generation through frameshift, support largely negative selection pressures related to frame disruption during gene evolution.

Data from this analysis is available on request from the author.

\section{Methods \\ Annotating disrupted mRNAs (dmRNA)}

An overview of the pipeline for disrupted mRNA (dmRNA) annotation is illustrated (Figure 5).

(i) Datasets and initial alignments: Gene annotations and genome sequence data for human build 35 were obtained from the Ensembl database. Human Refseq mRNAs and Unigene consensus sequences were also obtained from the NCBI. Any 3'-end polyadenylation was removed from these NCBI transcripts before alignment. These NCBI transcripts sequences were paired with Ensembl annotations from genomic DNA, using BLASTN [29] with e-value threshold of $\leq 10^{-10}$, sequence identity $\geq 98 \%$ and only considering alignment lengths $\geq 100$ nucleotides, that span $\geq$ a 0.99 fraction of each of the aligned sequences. Single-exon genes are discarded at this point.

(ii) Protein alignments to find frame disruptions: The UniProt/SwissProt protein database [25], was filtered to remove hypothetical proteins, and BLASTed against each of the Ensembl and NCBI transcript data sets, using evalue $\leq 10^{-4}$. The protein matches were filtered to pick the best-matching protein to each nucleotide sequence, at each position, as described previously [3]. These bestmatching proteins were then masked for low-complexity sequence using the program SEG [30], and then aligned to the nucleotide sequences a second time, using FASTX/Y (e-value threshold $\leq 0.01$ ) [31]. The extra SEG masking is necessary to remove false positives arising from a small number of repetitive protein sequences. If these second

Table 5: Frame disruption placement and nonsense-mediated decay

\begin{tabular}{|c|c|c|c|c|}
\hline \multirow[t]{2}{*}{ Type of initial frame disruption } & \multirow[t]{2}{*}{ Alignment verification } & \multicolumn{3}{|c|}{ NMD or non-NMD region * } \\
\hline & & $\begin{array}{l}\text { Observed } \\
\text { [NMD : non-NMD] }\end{array}$ & $\begin{array}{l}\text { Expected } \\
\text { [NMD : non-NMD] }\end{array}$ & Significance \\
\hline \multirow[t]{2}{*}{ Frameshift } & All cases & $159: 188$ & $294.9: 52.1$ & t+t \\
\hline & Cases with verifying alignments & $122: 159$ & $206.6: 74.4$ & tt† \\
\hline \multirow[t]{2}{*}{ Stop codon } & All cases & $43: 30$ & $42.9: 30.1$ & N.S. \\
\hline & Cases with verifying alignments & $22: 17$ & $17.5: 21.5$ & N.S. \\
\hline \multirow[t]{3}{*}{ Frameshift or stop codon } & All cases & $201: 218$ & $344.5: 74.5$ & $t+t$ \\
\hline & Cases with verifying alignments & $|4|: 184$ & $232.0: 93.0$ & t†t \\
\hline & $\begin{array}{l}\text { Cases with verifying alignments } \\
\text { (without 'probable UTR features') }\end{array}$ & $87: 122$ & $111.5: 97.5$ & t†t \\
\hline
\end{tabular}

* Ratios are for numbers expected or observed in NMD regions versus non-NMD ones. Expectations and tests are performed as for Table I. 


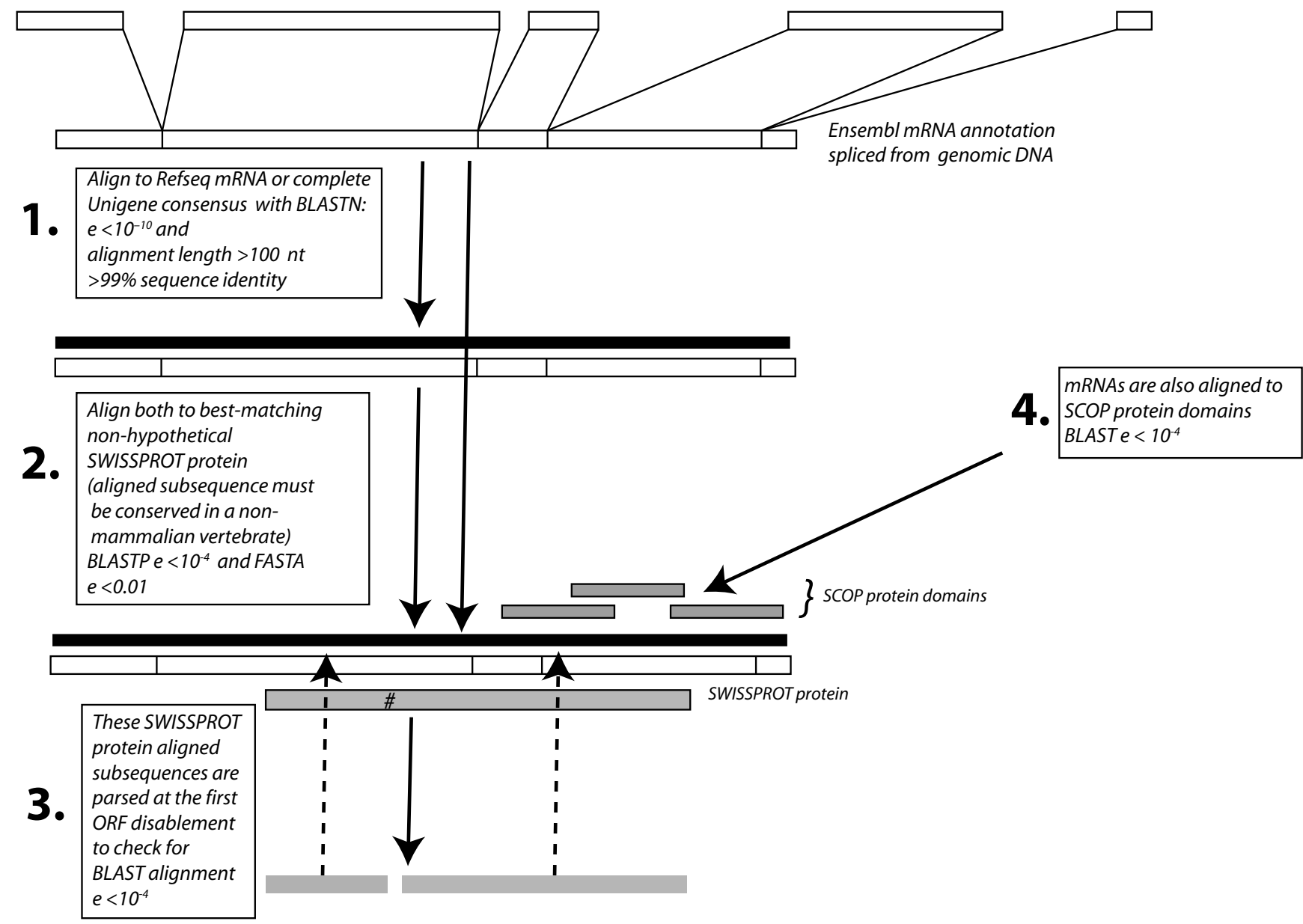

Figure 5

Pipeline for annotating dmRNAs. The steps discussed in Methods are illustrated schematically.

masked alignments proved significant, a final third FASTX/Y alignment was generated without masking in the sequences (with $\mathrm{e} \leq 0.01$ ).

Given that cDNAs may have error rates as high as as 1 in $1 \times 10^{-2}$, and that the genomic DNA error rate is $1 \times 10^{-4}$, we can expect that 1 in $1 \times 10^{-6}$ frame disruptions detected have arisen from sequencing error. Given, that we have a total of 729 frame disruptions in a total of 942,752 nucleotides, we would expect that, at most, only one of these frame disruptions has arisen from sequencing error. This implies that the data set of dmRNAs is of sufficiently high quality for in-depth bioinformatic analysis.

Alu elements are a common pollutant in protein databases [17]. We obtained a large number of matches to Alu elements producing dmRNAs; since these are not a focus of our current analysis, they were removed using proteinlevel translations of $A l u$ sequences, with a more accommodating BLAST threshold of $\mathrm{e} \leq 0.01$.
We removed selenoproteins from the dataset, since these are a known example of a re-coding phenomenon [22]. This was achieved through protein-level BLAST comparisons $\left(e \leq 10^{-4}\right)$ to the determined human selenoproteome, downloaded from the SelenoDB database [22].

To insure that we are not considering spurious frameshifts arising from bad protein annotations, we used an additional filter to insure that the protein reading frames in question are well conserved in a distant mammal or vertebrate. We required that the matching protein is conserved in a rodent or non-mammal vertebrate (with BLAST evalue $\geq 10^{-4}$ ) over $\geq 95 \%$ of its length.

(iii) Realignment to remove spurious frame disruptions: Spurious frame disruptions can arise as alignment errors when comparing a protein to a nucleotide sequence [3]. Such spurious frame disruptions are more frequent in more divergent aligned sequences; they are typically near the ends of aligned subsequences, and can also arise in 
compositionally-biased or low-complexity regions [3]. To insure that spurious frame disruptions are not considered in the present analysis, we parse the disrupted coding sequences at the initial frame disruption into two subsequences, and require that both of these subsequences align significantly to the original matching protein (BLAST e-value $\leq 10^{-4}$ ).

In addition, we checked for compensatory frameshifts (i.e., pairs of frameshifts that move a coding sequence out of frame, and then back into frame). It is possible that compensatory frameshifts provide a mechanism for generating sequence diversity in proteins over evolution. We checked for compensatory frameshifting, using an additional filter in the initial pipeline at step 3 (Figure 5). For every case of an initial frameshift, we checked for a second frameshift 3' to it in the transcript that corrects for the first frameshift. Then we checked whether the three subsequences delimited by these two frameshifts all align significantly with the original matching protein.

(iv) Protein domain matching: The Ensembl and NCBI transcripts were searched against the ASTRALSCOP protein domain database [27], using BLAST (e-value $\leq 10^{-4}$ ), and the best-matching domains at each position in a transcript were retained, as described previously $[8,3]$.

Specifically, also, we extracted zinc-finger motif assignments from the feature table records of the UniProt/ SWISSPROT database [25].

\section{Checking for evolution through frameshifting}

We checked for evidence of protein structure evolution through frameshift, using a modification of the initial pipeline (Figure 5). All steps were performed as before, except with SCOP protein domain sequences in lieu of SWISSPROT sequences. Then, we checked for any significant undisrupted matches to other protein domains at the same positions as these frameshifted protein domain matches, using a similar protocol of BLAST database searching, followed by refined alignment using FASTX/Y.

\section{Authors' contributions}

$\mathrm{PH}$ wrote the paper and performed most of the data analysis. ZY did some initial data analysis. Both authors have read and approved the final manuscript.

\section{Additional material}

\section{Additional file 1}

Supplementary Table 1: GO categories. The most abundant Gene Ontology (GO) functional categories for different data sets of exons are listed. Click here for file

[http://www.biomedcentral.com/content/supplementary/1471-

2164-8-371-S1.doc]

\section{Acknowledgements}

This work was supported by a Discovery grant to P.H. from the National Science and Engineering Research Council of Canada.

\section{References}

I. Harrison P, Gerstein M: Studying genomes through the aeons: protein families, pseudogenes and proteome evolution. J Mol Biol 2002, 3 I 8(5): II55-1 I74.

2. Harrison PM, Zheng D, Zhang Z, Carriero N, Gerstein M: Transcribed processed pseudogenes in the human genome: an intermediate form of expressed retrosequence lacking protein-coding ability. Nucleic acids research 2005, 33:2374-2383.

3. Harrison PM, Hegyi H, Balasubramanian S, Luscombe NM, Bertone P, Echols N, Johnson T, Gerstein M: Molecular fossils in the human genome: identification and analysis of the pseudogenes in chromosomes 2 I and 22. Genome Res 2002, I 2(2):272-280.

4. Esnault C, Maestre J, Heidmann T: Human LINE retrotransposons generate processed pseudogenes. Nature genetics 2000 , 24(4):363-367.

5. Karro JE, Yan Y, Zheng D, Zhang Z, Carriero N, Cayting P, Harrison $P$, Gerstein M: Pseudogene.org: a comprehensive database and comparison platform for pseudogene annotation. Nucleic acids research 2007, 35:D55-D60.

6. Yu Z, Morais D, Ivanga M, Harrison PM: Analysis of the role of retrotransposition in gene evolution in vertebrates. $B M C$ Bioinformatics 2007, 8:308.

7. Zhang Z, Harrison P, Liu Y, Gerstein M: Millions of years of evolution preserved: a comprehensive catalog of the processed pseudogenes in the human genome. Genome Res 2003, 13(I 2):254|-2558.

8. Zhang Z, Harrison P, Gerstein M: Identification and analysis of over 2000 ribosomal protein pseudogenes in the human genome. Genome Res 2002, I2(10): I466- | 4482

9. Vinckenbosch N, Dupanloup I, Kaessmann H: Evolutionary fate of retroposed gene copies in the human genome. Proc Natl Acad SCi USA 2006, I 03:3220-3225.

10. Modrek B, Lee CJ: Alternative splicing in the human, mouse and rat genomes is associated with an increased frequency of exon creation and/or loss. Nat Genet 2003, 34(2): 177-180.

II. Lewis BP, Green RE, Brenner SE: Evidence for the widespread coupling of alternative splicing and nonsense-mediated mRNA decay in humans. Proc Natl Acad Sci USA 2003, 100:189-192.

12. Letunic I, Copley RR, Bork P: Common exon duplication in animals and its role in alternative splicing. Hum Mol Genet 2002, II:I56I-I567.

13. Liang $H$, Landweber $L$ : A genome-wide study of dual coding regions in human alternatively spliced genes. Genome Res 2006, 16:190-196.

14. Raes J, Van de Peer Y: Functional divergence of proteins through frameshift mutations. Trends Genet 2005, $21: 428-431$.

15. Okamura K, Feuk L, Marques-Bonet T, Navarro A, Scherer SW: Frequent appearance of novel protein-coding sequences by frameshift translation. Genomics 2006, 88:690-697.

16. Frith MC, Wilming LG, Forrest A, Kawaji H, Tan SL, Wahlestedt C, BAjic VB, Kai C, Kawai J, Carninci P, Hayashizaki Y, Bailey TL, Huminiecki L: Pseudo-messenger RNA: phantoms of the transcriptome. PLoS Genet 2006, 2:e23. 
17. Sorek R, Ast G, Graur D: Alu-containing exons are alternatively spliced. Genome Res 2002, I2:1060-1070.

18. Maquat LE: Nonsense-mediated mRNA decay: splicing, translation and mRNP dynamics . Nat Rev Mol Cell Biol 2004, 5:89-99.

19. Ford AS, Guan Q, Neeno-Eckwall E, Culbertson MR: Ebs Ip, a negative regulator of gene expression controlled by the Upf proteins in the yeast Saccharomyces cerevisiae. Eukaryot Cell 2006, 5:30I-3I2.

20. Inoue K, Khajavi M, Ohyama T, Hirabayashi S, Wilson J, Reggin JD, Mancias P, Butler IJ, Wilkinson MF, Wegner M, Lupski JR: Molecular mechanism for distinct neurological phenotypes conveyed by allelic truncating mutations. Nat Genet 2004, 36:36I-369.

21. Korneev SA, Park JH, O'Shea M: Neuronal expression of neural nitric oxide synthase (nNOS) protein is suppressed by an antisense RNA transcribed from an NOS pseudogene. J Neurosci 1999, I 9(18):77| I-7720.

22. Fomenko DE, Xing W, Adair BM, Thomas DJ, Gladysher VN: Highthroughput identification of catalytic redox-active cysteine residues. Science 2007, 315:387-389.

23. Hamilton AT, Huntley S, Tran-Gyamfi M, Baggott DM, Gordon L, Stubbs L: Evolutionary expansion and divergence in the ZNF9I subfamily of primate-specific zinc finger genes. Genome Res 2006, 16:584-594.

24. Scofield DG, Hong $X$, Lynch M: Position of the final intron in fulllength transcripts: determined by NMD? Mol Biol Evol 2007 24:896-899.

25. Apweiler R, Bairoch A, Wu CH, Barker WC, Boeckmann B, Ferro S, Gasteiger E, Huang H, Lopez R, Magrane M, O'Donovan C, Redasch N, Yeh LS: UniProt: the Universal Protein knowledgebase. Nucleic acids research 2004, 32:DII5-DII9.

26. Lareau LF, Inada M, Green RE, Wengrod JC, Brenner SE: Unproductive splicing of SR genes associated with highly conserved and ultraconserved DNA elements. Nature 2007, e-pub :.

27. Chandonia JM, Hon G, Walker NS, Lo Conte L, Koehl P, Levitt M, Brenner SE: The ASTRAL Compendium in 2004. Nucleic acids research 2004, 32:DI89-DI92.

28. Mendell JT, Sharif NA, Meyers J, Martinez F, Dietz HC: Nonsense surveillance regulates expression of diverse classes of mammalian transcripts and mutes genomic noise. Nat Genet 2004, 36:1073-1078.

29. Altschul SF, Madden TL, Schaffer AA, Zhang J, Zhang Z, Miller W, Lipman DJ: Gapped BLAST and PSI-BLAST: a new generation of protein database search programs. Nucleic acids research 1997, 25(I7):3389-3402.

30. Wootton JC, Federhen S: Analysis of compositionally biased regions in sequence databases. Methods Enzymol 1996, 266:554-57I.

31. Pearson WR: Flexible sequence similarity searching with the FASTA3 program package. Methods Mol Biol 2000, 132:185-219.
Publish with Biomed Central and every scientist can read your work free of charge

"BioMed Central will be the most significant development for disseminating the results of biomedical research in our lifetime. "

Sir Paul Nurse, Cancer Research UK

Your research papers will be:

- available free of charge to the entire biomedical community

- peer reviewed and published immediately upon acceptance

- cited in PubMed and archived on PubMed Central

- yours - you keep the copyright
BioMedcentral 